“ (C) 2017 IEEE. Personal use of this material is permitted. Permission from IEEE must be obtained for all other uses, in any current or future media, including

reprinting/republishing this material for advertising or promotional purposes, creating new collective works, for resale or redistribution to servers or lists, or reuse of any copyrighted component of this work in other works." 


\section{Gaussian Process Model Enabled Particle Filter for Device-Free Localization}

\author{
Biao Song, Hailong Wang, Wendong Xiao \\ Beijing Engineering Research Center of Industrial \\ Spectrum Imaging, School of Automation \& Electrical \\ Engineering \\ University of Science and Technology Beijing, \\ Beijing, China \\ \{songbiao7664,whl890811\}@163.com; \\ wdxiao@ustb.edu.cn
}

\author{
Shoudong Huang, Lei Shi \\ Centre for Autonomous Systems, \\ Faculty of Engineering and Information Technology \\ University of Technology Sydney \\ Sydney, Australia \\ \{Shoudong.Huang, Lei.Shi-1\}@uts.edu.au
}

\begin{abstract}
Device-free localization (DFL) is an emerging wireless network target localization technique that does not need to attach any electronic device with the target. It is remaining as a challenging research problem due to the weak wireless signals and the uncertain wireless communication environment. In this paper, a novel Gaussian Process (GP) based wireless propagation model is proposed to describe the likelihood relationship between the target location and the changes of the RSS measurement for a wireless link. Sequentially Particle Filter (PF) is applied to the DFL for estimating the location of the target, after the GP model is trained using the experimental measurements of the link. Experimental results demonstrate that the proposed GP-PF algorithm can track the target with much better localization accuracy than the Support Vector Machine (SVM) based PF approach.
\end{abstract}

Keywords-Device-free localization (DFL); Gaussian Process (GP); Particle Filter (PF)

\section{INTRODUCTION}

Device-free localization (DFL) is receiving tremendous interests for target localization without attaching any device with the target. In a DFL system, a number of signal transmitters (RTs) and signal receivers (RXs) are deployed. It is found that, when the target enters the monitoring area, the received signal strength (RSS) measurements of certain links between the RTs and RXs will change significantly, and can be used to estimate the target location [1]. DFL has found a lot of applications such as medical healthcare, security safeguard, and emergency rescue.

Due to the weak wireless signals and the uncertain and dynamic wireless propagation environment suffering from nonline-of-sight, multi-path, and fading phenomena, accurate DFL is remaining as a challenging research problem and a lot of research work has been done in the recent years. K.E. Kafrawy et al. [2] investigated the impact of the human motion on the changes of the RSS measurement of the link. Youssef et al., proposed a fingerprinting approach for Wi-Fi-based DFL [3][5]. The fingerprinting approach consists two stages, an offline stage and an online stage. During the offline stage, the RSS measurements of links were collected when the target is located at certain reference points with known locations, and the offline radio map was built to record the locations of the reference points and their associated differential RSS values between the RSS measurements of links with and without the target. During the online stage, the real-time differential RSS measurements of links were compared with the offline radio map to estimate the target location. As a must, in this approach, tedious calibration procedure is required. It is also challenging to efficiently build and store the radio map. If the wireless network is changed, the offline radio map need to be renewed accordingly which may not be so easy in the real situation.

Motivated by X-ray tomography, Wilson, J. and Patwari, N. proposed the RTI approach based on the differential RSS measurements [6]-[8] via the reconstruction of the tomography image for the locations of the target, and formulate the DFL problem as a linear ill-posed inverse problem, then solve it by the regularization method. Kaltiokallio, O. et al. presented an on-line recalibration method that allows the system to adapt to the changes in the radio environment [9]. The performance of those methods can be improved with the assistance of the frequency diversity [10]. Wang, J. et al. proposed the compressive sensing approach to deal with the space-domain sparse information to tackle the ill-posed inverse problem in signal reconstruction [11]-[13].

To reduce the computation overhead in solving the illposed inverse problem, Bayesian grid approach (BGA) was proposed in [14] by utilizing the observation information of the shadowed links, the prior estimation information, and the constraint information of the non-shadowed links. Savazzi, S. et al. proposed a joint model based on the theory of diffraction to deal with the average path loss and the fluctuations of the RSS measurements induced by the moving target, and derive a novel stochastic Bayesian model for the real-time estimation of the target location [15].

D. Zhang et al. [16], [17] proposed geometrical methods based on the influential links. J. Wang, et al. [18] proposed an elliptical model (EM) and the method to estimate the states of links (including: affected link and outlier link). W. Xiao, et al. proposed the nonlinear optimization model and the outlier rejection method based on the geometrical positional relationship among links [19]. Y. Gou [20] proposed the exponential-Rayleigh model (ERM) in which the Rayleigh 
term is added to compensate the RSS. This model is still not accurate enough to model the RSS variation when the target is located in the proximity of a LOS path. Z. Wang et al. proposed the Diffraction Measurement model (DM) which invokes diffraction theory and regards the target as a cylinder instead of a point mass [21]. DM is highly nonlinear with high computation complexity.

Gaussian Process (GP) is a non-parametric and flexible model [21-23] that may be suitable for DFL and needs for further theoretic and experimental studies. Therefore the GP enabled Particle Filter (GP-PF) algorithm is proposed for DFL in this paper. The signal propagation model is trained by the GP model as the observation model for the target tracking under the PF framework.

The paper is organized as follows: the theoretical background on GP is introduced in Section II; the GP based wireless propagation model is proposed in Section III. PF method for DFL is presented in Section IV. Experimental evaluation results are reported in Section V. Finally, the conclusions and the future work are given.

\section{THEORETICAL BACKGROUND}

GP can learn regression function from training data, and provide uncertainty estimates. There are several ways to interpret GP regression models, and we use the function-space view to describe GP [22].

Definition 1. A Gaussian process is a collection of random variables, any finite number of which have a joint Gaussian distribution.

Let us assume the training data and the testing data are $D=\left\{\left(\mathrm{x}_{1}, \mathrm{y}_{1}\right),\left(\mathrm{x}_{2}, \mathrm{y}_{2}\right), \ldots,\left(\mathrm{x}_{m}, \mathrm{y}_{m}\right)\right\}$ and $T=\left\{\left(\mathrm{x}_{1}^{*}, \mathrm{y}_{1}^{*}\right),\left(\mathrm{x}_{2}^{*}, \mathrm{y}_{2}^{*}\right), \ldots,(\right.$

$\left.\left.\mathrm{x}_{m}^{*}, \mathrm{y}_{m}^{*}\right)\right\}$, respectively. The observation function is

$$
y=f(x)+\varepsilon
$$

where $x$ is the n-dimensional input vector, $y$ is the observed value, or the output sample in $R$, and $\varepsilon$ is a generalized additive independent Gaussian distribution $N\left(0, \sigma_{i}^{2}\right)$ with the known variance $\sigma_{i}^{2}$. For the sake of simplicity, let the $n \times m$ dimensional matrix $X$ denote the training input, the $\mathrm{m}$ dimensional vector $y=\left\{\mathrm{y}_{1}, \mathrm{y}_{2}, \ldots, \mathrm{y}_{m}\right\}$ denote the training output.

In GP, the observation function $f$ can be described by the mean function $m(x)$ and the covariance function $k\left(x, x^{*}\right)$ :

$$
m(x)=E[\mathrm{f}(\mathrm{x})]
$$

$$
k\left(x, x^{*}\right)=E\left[(\mathrm{f}(\mathrm{x})-E[\mathrm{f}(\mathrm{x})])\left(\mathrm{f}\left(\mathrm{x}^{*}-E\left[\mathrm{f}\left(\mathrm{x}^{*}\right)\right]\right)\right)\right] .
$$

The choice of the covariance function usually depends on the user. In this paper, the following widely used squared exponential (SE) covariance function is selected

$$
\operatorname{cov}\left(f\left(\mathrm{x}_{i}\right), f\left(\mathrm{x}_{j}\right)\right)=k\left(\mathrm{x}_{i}, \mathrm{x}_{j}\right)=\exp \left(-\frac{\left|\mathrm{x}_{i}-\mathrm{x}_{j}\right|^{2}}{2}\right)
$$

As this covariance function is infinitely differentiable and smooth.

In the observation function, the noise $\varepsilon$ is additive independent Gaussian distributed with the variance $\sigma_{i}^{2}$, the corresponding covariance function on the noisy observations is

$$
\operatorname{cov}\left(\mathrm{y}_{i}, y_{j}\right)=k\left(\mathrm{x}_{i}, \mathrm{x}_{j}\right)+\sigma_{i}^{2} \delta_{i j}
$$

where $\delta_{i j}$ is a Kronecker delta. If $i=j, \delta_{i j}=1$, and otherwise $\delta_{i j}=0$. The joint distribution of the observed values y (the training data output), and the function values $\mathrm{y}^{*}$ (the test data output) can be written as

$$
\left[\begin{array}{c}
\mathrm{y} \\
\mathrm{y}^{*}
\end{array}\right] \sim N\left[\begin{array}{cc}
K(X, X)+\sigma_{m}^{2} I_{m \times m} & K\left(X, X_{*}\right) \\
K\left(X_{*}, X\right) & K\left(X_{*}, X_{*}\right)
\end{array}\right],
$$

where $X$ is the $n \times m$ design matrix of the aggregated training input vectors $x, X_{*}$ is the $n \times m^{*}$ matrix of the aggregated test input vectors $x^{*}, I_{m \times m}$ is the identity matrix, and $\sigma_{m}^{2}$ is the variance of the observation noise.

Then the predictive condition distribution $p\left(y^{*} \mid X, y, x^{*}\right)$ for the single test output can be derived as

$$
\begin{gathered}
p\left(y^{*} \mid X, y, x^{*}\right) \sim N(\mu, \sigma) \\
\mu=\bar{f}_{*}=k_{*}^{T}\left[K(X, X)+\sigma_{m}^{2} I_{m \times m}\right]^{-1} y \\
\sigma=\operatorname{var}\left(f_{*}\right)=k\left(\mathrm{x}_{*}, \mathrm{x}_{*}\right)-k_{*}^{T}\left[K(X, X)+\sigma_{m}^{2} I_{m \times m}\right]^{-1} k_{*}
\end{gathered}
$$

where $k_{*}$ is $k\left(X, \mathrm{x}_{*}\right)$ which is the covariance vector. Here we rewrite the mean function as

$$
\bar{f}_{*}=\sum_{i=1}^{m} \alpha_{i} k\left(x_{i}, x_{*}\right)
$$

where $\alpha_{i}$ is $\left[K(X, X)+\sigma_{m}^{2} I_{m \times m}\right]^{-1} y$. It indicates that the mean function is a linear combination of training data output $y$. The covariance function can be estimated by the training data and the input of the test data. 


\section{MODEL FORMULATION}

In this section, the GP wireless signal propagation model will be presented.

Suppose there are $\mathrm{M}$ wireless nodes with known locations in the sensor network. Let $L_{n}(i, j)$ denotes the ID of the link $n$ between the wireless node $i$ and the wireless node $j, \operatorname{dis}_{n}(i, j)$ denotes the distance between the wireless node $i$ and the wireless node $j, R S S_{n}^{t}$ denotes the RSS measurement of link $n$ at time $\mathrm{t}$.

The wireless signal is sensitive to the uncertain communication environment, and the noises are hard to estimate. In this paper, the signal propagation model with incorporating the uncertain noise will be built by GP.

Normally the signal propagation model is used to describe the relationship between differential RSS and the relative positional information among the target, the transmission node (RT) and the receiving node $(\mathrm{RX}) \Delta d$, i.e., $\Delta R S S=f(\Delta d)$. As showed in Fig. 1, as the key of those models, $\Delta d$ is defined as

$$
\Delta d=d_{R T}+d_{R X}-d
$$

where $d_{R T}$ is the distance between the target and RT, $d_{R X}$ is the distance between the target and TX, and $d$ is the length of the link.

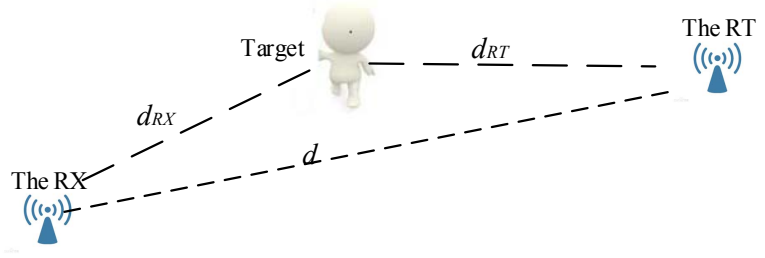

Figure 1 the positional relationship between the target and the wireless nodes

It is clear that the differential RSS of the link is caused by the relative location between the target and the wireless nodes $X_{w n}\left(\mathrm{x}_{i}, \mathrm{y}_{i}\right)$ (the $\mathrm{RT}$ and the $\left.\mathrm{RX}\right)$ and the distance of the link $\operatorname{dis}_{n}(i, j)$. Here, we assume the function $f$ that decries the signal propagation model is

$$
\Delta R S S_{n}^{t}=f\left(X, X_{w n}\left(\mathrm{x}_{i}, \mathrm{y}_{i}\right), d i S_{n}(i, j)\right)+\eta_{n},
$$

where $\eta_{\mathrm{n}}$ is the noise for link n. For simplification, we assume that RT is in the coordinate origin, and RX is in the $\mathrm{x}$-axis in the Cartesian coordinate system. As showed in Fig. 2, the target location can be transformed into the relative coordinates $X_{n}\left(\mathrm{x}_{i}, \mathrm{y}_{i}\right)$ for link $\mathrm{n}$. We can rewrite (9) as

$$
R S S_{n}^{t}=f\left(X_{n}\left(\mathrm{x}_{i}, \mathrm{y}_{i}\right), \operatorname{dis}_{n}(i, j)\right)+\eta_{n}
$$

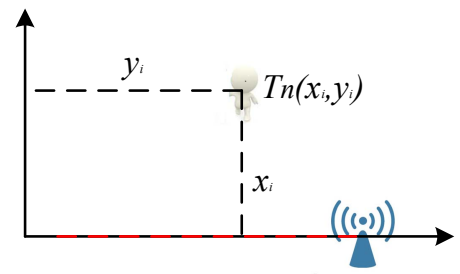

Figure 2 the relative coordinates for the link

The wireless signal is easy to be disturbed by the external environment and the interference factors, and it is hard to estimate the noise $\eta_{n}$. As the training data is collected by the experiment, and the noise caused by the external environment is included in the training data, the noise can be regard as the part of the GP signal propagation model. We can rewrite the GP model as

$$
\Delta R S S_{n}^{t}=g\left(X_{n}\left(\mathrm{x}_{i}, \mathrm{y}_{i}\right), d i s_{n}(i, j)\right)+\varepsilon
$$

where $\varepsilon$ is the additive independent Gaussian distribution $N\left(0, \sigma_{i}^{2}\right)$ to compensate the uncertain RSS variation.

The format of the training data used in this paper is different from that used in the past radio map for the DFL. For the radio map of the fingerprinting approach, the whole network and some reference points are set up, and $R S S=\left\{\left(R S S_{1}^{1}, \ldots, R S S_{n}^{1}\right), \ldots,\left(R S S_{1}^{t}, \ldots, R S S_{n}^{t}\right)\right\} \quad$ and the target location are recorded.

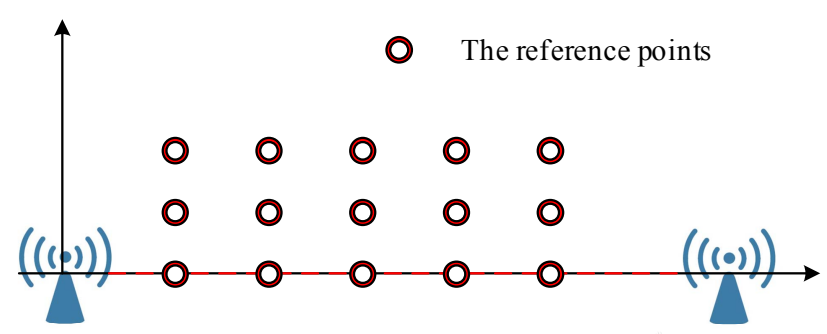

Figure 3 the reference points

As shown in Fig.3, one wireless link with a transmission node and a receiving node is selected, and some points around the link are used as the reference points for the training data. The reference point location $X_{i}\left(\mathrm{x}_{i}, \mathrm{y}_{i}\right)$ and the distance of the link $d i s_{n}$ are the input, and the $R S S_{i}$ is the output of the GP model. Hence, the set of the training data is

$$
\operatorname{Tr}=\left\{\left(\mathrm{x}_{1}, \mathrm{y}_{1}, d i s_{1}, \Delta R S S_{1}\right), \ldots,\left(\mathrm{x}_{r}, \mathrm{y}_{r}, d i s_{r}, \Delta R S S_{r}\right)\right\},
$$

where the number of the train data is $r$. 
Let $T r_{\text {input }}=\left\{\left(\mathrm{x}_{1}, \mathrm{y}_{1}, d i s_{1}\right), \ldots,\left(\mathrm{x}_{r}, \mathrm{y}_{r}, d i s_{r}\right)\right\}$ and $T r_{\text {output }}=\{$ $\left.\Delta R S S_{1}, \ldots, \Delta R S S_{r \times n}\right\}$ denote the input and output of the training data, and $T e_{\text {input }}^{t}=\left\{\left(\mathrm{x}_{t}, \mathrm{y}_{t}, d i s_{i}^{t}\right)\right\}$ and $T e_{\text {output }}^{t}=\left\{R S S_{i}^{t}\right\}$ is the input and output for the test location respectively. According to eq. (7), (11), the predictive condition distribution for the presence of the target at time t $p\left(T e_{\text {output }}^{t} \mid \operatorname{Tr}_{\text {input }}, T r_{\text {output }}, T e_{\text {input }}^{t}\right)$ is

$$
\begin{gathered}
p\left(T e_{\text {output }}^{t} \mid T r_{\text {input }}, T r_{\text {output }}, T e_{\text {input }}^{t}\right) \sim N(\mu, \sigma) \\
\mu=k_{*}^{T}\left[K\left(\operatorname{Tr}_{\text {input }}, \operatorname{Tr}_{\text {input }}\right)+\sigma_{m}^{2} I_{m \times m}\right]^{-1} T r_{\text {output }} \\
\sigma=k\left(T e_{\text {input }}^{t}, T e_{\text {input }}^{t}\right)-k_{*}^{T}\left[K\left(T r_{\text {input }}, T r_{\text {input }}\right)+\sigma_{m}^{2} I_{m \times m}\right]^{-1} k_{*}
\end{gathered}
$$

where the SE covariance function is selected. $\mu$ and $\sigma$ are the mean and variance of the RSS measurements of link $i$ according to the GP model for the signal propagation, respectively.

\section{TARGET TRACKING BASED ON PARTICLE FILETER}

\section{A. Motion Model}

In this paper, the target state $X_{t}$ for time step $t$ is defined as $\left[x_{t}, \dot{x}_{t}, y_{t}, \dot{y}_{t}\right]$, including the location $\left(x_{t}, y_{t}\right)$ and the velocity $\left(\dot{x}_{t}, \dot{y}_{t}\right)$ of the target. The following constant velocity target motion model is used

$$
X_{t}=F X_{t-1}+\varepsilon_{t}
$$

where $X_{t-1}$ is the state for time step $t-1$, and $\varepsilon_{t}$ is the Gaussian noise.

\section{B. Particle Filter}

Due to the excellent performance of Kalman Filter (KF) and Particle Filter (PF), they have been widely used in the target localization and tracking. PF is a sequential Monte Carlo method calculates the posterior probability distribution function (PDF) based on a set of the weighted particles. As it can solve the complex nonlinear problem without the Gaussian conditions, which is the case in this paper, PF will be used to track the target in the DFL.

Let us assume the set of all the measurements for the links till time step $\mathrm{t}$ is

$$
z_{1, \ldots, t}=\left\{\mathrm{z}_{1,} \mathrm{z}_{2}, \ldots, \mathrm{z}_{t}\right\}
$$

where $\mathrm{z}_{i}$ is $\left\{\mathrm{z}_{i}^{1}, \ldots \mathrm{z}_{i}^{n}\right\}$. The Bayesian based the maximal posterior distribution $p\left(X_{t} \mid z_{1, \ldots, t}\right)$ is used to estimate the target location, i.e., $\hat{X}_{t}=\underset{X_{t}}{\arg \max } p\left(X_{t} \mid z_{1, \ldots, t}\right)$. The posterior PDF can be acquired by the prediction and measurement update as follows:

$$
\begin{gathered}
p\left(X_{t} \mid z_{1, \ldots, t-1}\right)=\int p\left(X_{t} \mid z_{1, \ldots, t-1}\right) p\left(X_{t} \mid X_{t-1}\right) \mathrm{d} X_{t-1} \\
p\left(X_{t} \mid z_{1, \ldots, t}\right)=\frac{p\left(X_{t} \mid z_{t}\right) p\left(X_{t} \mid z_{1, \ldots, t-1}\right)}{p\left(z_{t} \mid z_{1: t-1}\right)}
\end{gathered}
$$

Assume the number of the particles is $N_{p}$ at the current time $t$ and $X_{t}^{i}$ is the state of the particle $\mathrm{i}$ with the weight $\omega_{t}^{i}$. The posterior PDF $p\left(X_{t} \mid z_{1, \ldots, t}\right)$ can be approximated by the $\mathrm{PF}$ algorithm with a set of weighted particles:

$$
p\left(X_{t} \mid z_{1, \ldots, t}\right) \approx \sum_{i=1}^{N_{p}} \omega_{t}^{i} \delta\left(X_{t}-X_{t}^{i}\right)
$$

where $\delta$ is the proposal distribution, $p\left(X_{t}^{i} \mid X_{t-1}^{i}\right)$ is the transitional prior state. As it is difficult to build the optimal proposal distribution, we consider the proposal distribution is the transitional prior state. The weight of the particle can be recalculated by

$$
\omega_{t}^{i} \propto \omega_{t-1}^{i} p\left(z_{t} \mid X_{t}^{i}\right) \propto \omega_{t-1}^{i} \prod_{l=1}^{n} \exp \left(-\frac{\left[\Delta R S S_{t}^{i}\left(X_{t}^{i}, d i s_{n}\right)-z_{l}\right]^{2}}{2 \sigma^{2}}\right)
$$

where $R S S_{t}^{i}\left(X_{t}^{i}, d i s_{n}\right)$ is RSS measurement for the $i^{\text {th }}$ particle according to (13).

In order to guarantee the sum of the weights is 1 , the normalization of the weights is necessary, and can be performed as follows:

$$
\bar{\omega}_{t}^{i}=\omega_{t}^{i} / \sum_{j=1}^{N_{p}} \omega_{t}^{j}
$$

Then the target location can be estimated as $\hat{X}_{t}\left(\hat{x}_{t}, \hat{y}_{t}\right)=\sum_{i=1}^{N_{p}} \bar{\omega}_{t}^{i} \times X_{t}^{i}$.

The degeneration is one problem for the PF. In order to prevent this problem, the predefined threshold $N_{t h}$ is set. The number of the effective particles $N_{\text {eff }}$ is

$$
N_{e f f}=1 / \sum_{i=1}^{N_{p}}\left(\omega_{t}^{i}\right)^{2}
$$

If $N_{\text {eff }} \succ N_{\text {th }}$, the particles are in degeneration, and resampling is necessary to overcome it. 


\section{EXPERIMENTAL EVALUATION}

In this section, we will evaluate the GP-PF algorithm, and compare it with the SVM-PF via experiments.

\section{A. Experimental Setup}

The experimental system is set up in front of the main building of the University of Science and Technology Beijing. As shown in Fig. 4, the size of the monitoring area is $6 m \times 6 m$, and there is no construction in the monitoring area. The target is a person with the height $187 \mathrm{~cm}$ and the weight $90 \mathrm{~kg}$ moving with the speed $1 \mathrm{~m} / \mathrm{s}$. 16 TI CC2530 wireless nodes are uniformly distributed along the boundary of the monitoring area.

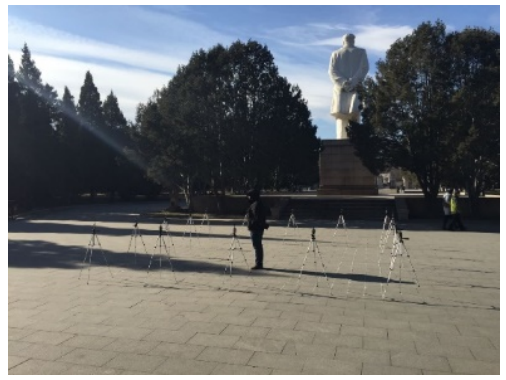

Figure 4 the test-bed set up

The IEEE 802.15.4 standard is used for the communication, and a simpler communications protocol is used to avoid the collision of the network. Each sensor has a unique ID. When one broadcasts the signal, others receive. One loop for the wireless nodes is $240 \mathrm{~ms}$ which is enough to track the target.

The following metric for the performance evaluation is error $_{i}$, which is the Euclidean distance between the target location and the estimated location,

$$
\text { error }_{i}=\sqrt{\left(\hat{x}_{t}-x_{t}\right)^{2}+\left(\hat{y}_{t}-y_{t}\right)^{2}}
$$

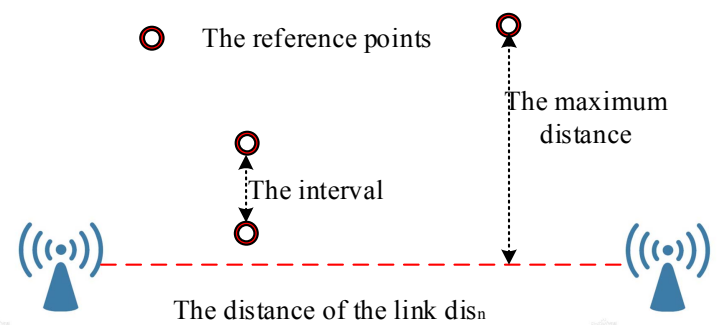

Figure 5 the distance between the reference point and the link

In our test-bed, the shortest and the longest distance of the link are $1.414 \mathrm{~m}$ and $8.4852 \mathrm{~m}$, respectively. The distance of the link $d i s_{n}=\{2 m, 3 m, 4 m, 5 m, 6 m, 7 m, 8 m\}$ are selected for the training data. The interval for the reference point is $0.2 \mathrm{~m}$, and the maximum distance between the reference point and the link is $0.6 \mathrm{~m}$, as it is shown in Fig. 5. The RSS measurements for a reference point are the average of 100 repeated times.

\section{B. Performance Evaluation}

As shown in Fig.6, the trajectory 'zigzag' is selected to compare GP-PF and SVM-PF. The initial particles is generated randomly in the monitoring area, and the number of the particle $N_{p}$ is 800 . The average result which repeats the PF for 100 times to compensate the random initial particles is used to evaluate the performance.

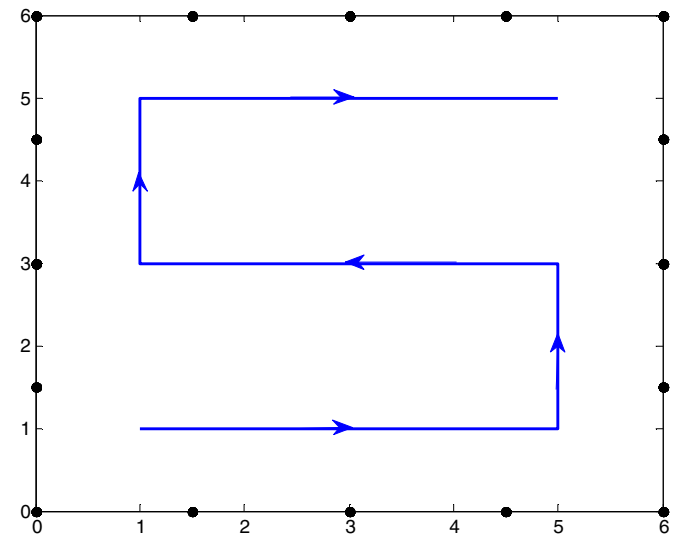

Figure 6 the trajectory of the target

The tracking results for GP-PF and SVM-PF is shown in Fig. 7 . The trajectory based on GP-PF is smoother SVM-PF. The details of the results are shown in the Table 2. The mean accuracy of GP-FP has increased 31\% than SVM-PF.

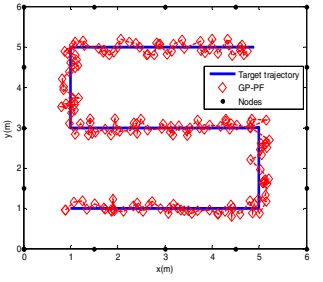

(a)

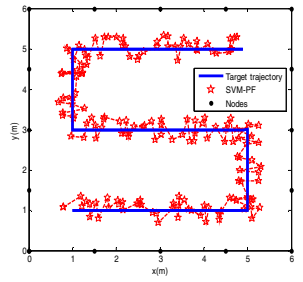

(b)
Figure 7

Table 2: The detail of the results between GP-PF and SVM-PF

\begin{tabular}{|c|c|c|}
\hline Method & Median $(\mathrm{m})$ & Mean $(\mathrm{m})$ \\
\hline GP-PF & 0.1319 & 0.1353 \\
\hline SVM-PF & 0.1995 & 0.1966 \\
\hline
\end{tabular}

The results of the CDF for the tracking errors are shown in Fig. 8 and Table 3 . It is clear that $100 \%$ of the tracking error for GP-PF is lower than $0.3 \mathrm{~m}, 29.38 \%$ and $16.25 \%$ is lower than $0.1 \mathrm{~m}, 85.62 \%$ and $50.62 \%$ is lower than $0.2 \mathrm{~m}$, for GP-PF and SVM-PF, respectively.

It is obviously that GP-PF is better than SVM-PF under this situation. The GP is suitable to build the signal propagation model. 


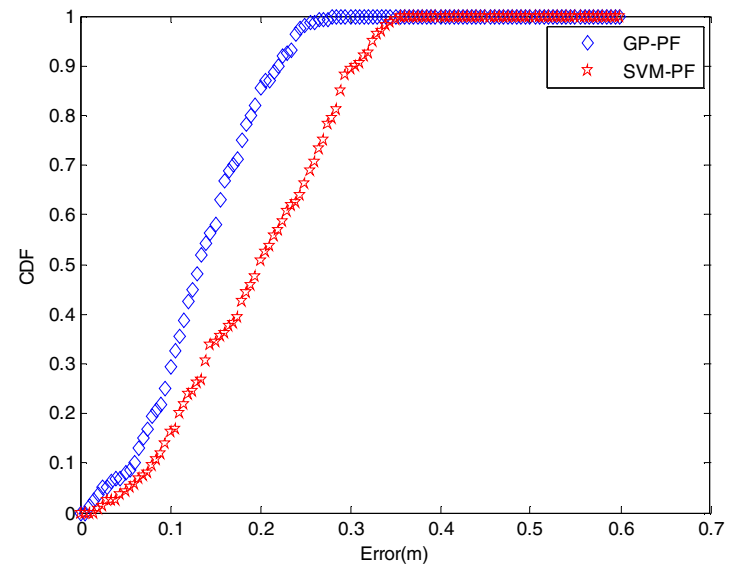

Figure 8 The CDF of the tracking error between GP-PF and SVM-PF

Table 3: The CDF of the tracking error between GP-PF and SVM-PF

\begin{tabular}{|c|c|c|c|}
\hline Accuracy & $0.1(\mathrm{~m})$ & $0.2(\mathrm{~m})$ & $0.3(\mathrm{~m})$ \\
\hline GP-PF & $29.38 \%$ & $85.62 \%$ & $100 \%$ \\
\hline SVM-PF & $16.25 \%$ & $50.62 \%$ & $89.38 \%$ \\
\hline
\end{tabular}

\section{CONCLUSIONS}

In this paper, the GP model which describes the likelihood relationship between the target location and the differential RSS is proposed. The GP model enabled PF algorithm is verified by the experiments for the DFL. For the GP model, the training data is built according to the size of the monitoring area. In the experiment, the size of the monitoring area is 36 square meter with 16 sensors. The mean accuracy of the GP model for the target tracking is $0.1353 \mathrm{~m}$. The GP model can provide the better accuracy than the SVM model. In the future, we will investigate the robustness of the localization algorithm under the variation of the environment, the problem of target tracking, and the localization and tracking of multiple targets.

\section{REFERENCES}

[1] M. Youssef, M. Mah, and A. Agrawala, "Challenges: Device-Free Passive Localization for Wireless Environments," Proc. 13th ACM MobiCom, Montreal, Canada, pp. 222-229, Sep. 2007.

[2] K.E. Kafrawy, M. Youssef, A.E. Keyi, "Impact of the Human Motion on the Variance of the Received Signal Strength of Wireless Links," Proc 22nd IEEE PIMRC, Toronto, Canada, pp. 1208-1212, Sept. 2011.

[3] Sabek, I.; Youssef, M. Multi-Entity Device-Free WLAN localization. In Proceedings of the IEEE Global Communications Conference, Anaheim, CA, USA, 3-7 December 2012, pp. 2018-2023..

[4] I. Sabek, M.Youssef, A.V. Vasilakos, "ACE: An Accurate and Efficient Multi-Entity Device-Free WLAN Localization System," IEEE Transactions on Mobile Computing, vol. 14, no. 2, pp. 621-632, Feb. 2015.

[5] M. Seifieldin, A. Saeed, A. E. Kosba, A. E. Keyi, M. Youssef, "Nuzzer: A Large-Scale Device-Free Passive Localization System for Wireless
Environments," IEEE Transactions on Mobile Computing, vol.12, no.17, pp. 1321-1334, Jul. 2013.

[6] J. Wilson, N. Patwari, "Radio tomographic imaging with wireless networks," IEEE Transactions on Mobile Computing, vol. 9, no. 5, pp. 621-632, May 2010.

[7] J. Wilson, N. Patwari, "See through walls: motion tracking using variance-based radio tomography networks," IEEE Transactions on Mobile Computing, vol. 10, no. 5, pp. 612-621, May 2011.

[8] Y., Zhao, N. Patwari, J.M. Phillips, "“"Radio Tomographic Imaging and Tracking of Stationary and Moving People via Kernel Distance," Proc. 12th ACM/IEEE IPIN, Philadelphia, USA, pp. 229-240, Apr. 2013.

[9] O. Kaltiokallio, M. Bocca, N. Patwari, "Follow @ grandma: Long-Term Device-Free Localization for Residential Monitoring," Proc.37th IEEE LCN Workshops, pp. 991-998, Oct. 2012.

[10] J. Wilson, N. Patwari, “A fade level skew-Laplace signal strength model for device-free localization with wireless networks," IEEE Transactions on Mobile Computing, vol. 11, no. 6, pp. 947-958, Jun. 2012.

[11] J. Wang, Q. Gao, X. Zhang, H. Wang, "Device-free localisation with wireless networks based on compressive sensing," IET Communications vol. 6, no. 15, pp. 2395-2403, Oct. 2012.

[12] J. Wang, Q. Gao, H. Wang, Y. Yu, M. Jin, "Time-of-Flight-Based Radio Tomography for Device Free Localization," IEEE Transactions on Wireless Communications, vol. 12, no. 5, pp. 2355-2365, Apr. 2013.

[13] J. Wang, D.Y. Fang, X.J. Chen, Z. Yang, T.Z. Xing, L. Cai, "LCS: Compressive Sensing based Device-Free Localization for Multiple Targets in Sensor Networks," Proc 2013 IEEE Infocom, Turin, Italy, pp. 145-149, Apr. 2013.

[14] J. Wang, Q. Gao, P. Cheng, L. Wu, K. Xin, H. Wang, "Lightweight Robust Device-Free Localization in Wireless Networks," IEEE Transactions on Industrial Electronics, vol. 61, no. 10, pp. 5681-5689, Jan. 2014.

[15] S. Savazzi, M. Nicoli, F. Carminati, M. Riva, "A Bayesian Approach to Device-Free Localization: Modeling and Experimental Assessment," IEEE Journal of Selected Topics in Signal Processing, vol. 8, no. 1, pp. 16-29, Oct.. 2014.

[16] D. Zhang, J. Ma, Q. Chen, and L.M. Ni, "An RF-based system for tracking transceiver-free objects," Proc. 15th IEEE PerCom, pp. 135144, Mar. 2007.

[17] D. Zhang, Y., H. Liu, X. N. Guo, L.M. Ni, "Fine-Grained Localization for Multiple Transceiver-Free Objects by using RF-Based Technologies" IEEE Transactions on Parallel And Distributed Systems vol. 25, no. 6, pp. 1464-1475, Jun. 2014.

[18] J. Wang, Q.H. Gao, Y. Yu, P. Cheng, L.F. Wu, H.Y. Wang, "Robust Device-Free Wireless Localization Based on Differential RSS Measurements," IEEE Transactions on Industrial Electronicsm, vol. 60, no. 12, pp. 5943-5952, Dec. 2013.

[19] W.D. Xiao, B. Song, X.T. Yu, P.Y. Chen, "Nonliner Optimization based Device-Free Localization with Outlier Link Rejection," Sensors 2015, 15(4), 8072-8087; doi:10.3390/s150408072.

[20] Y. Guo, K. Huang, N.Y. Jiang, X.M. Guo, Y.F. Li, G.L. Wang, “An Exponential-Rayleigh Model for RSS-Based Device-Free Localization and Tracking," IEEE Transactions on Mobile Computing, vol. 14, no. 3, pp. 484-494, Jun. 2014.

[21] Z.H. Wang, H. Liu, S.X, Xu, X.Y. Bu, J.P. An, “A Diffraction Measurement Model and Particle Filter Tracking Method for RSS-based DFL," IEEE Journal of Selected Topics in Signal Processing, vol. 33, no. 3, pp. 2391-2403, Nov. 2014.

[22] C. E. Rasmussen and C. K. I. Williams, "Gaussian Processes for Machine Learning," Cambridge, MA, USA: MIT Press, 2005.

[23] B Ferris, D Hähnel, D Fox, "Gaussian Processes for Signal StrengthBased Location Estimation," Proc.2006 Robotics Science and Systems, Pennsylvania, USA, Aug. 2006 